Noname manuscript No.

(will be inserted by the editor)

\title{
Resilient BBU Placement in 5G C-RAN over Optical Aggregation Networks
}

\author{
Mohamed Shehata ${ }^{1} 2^{*}$. Francesco \\ Musumeci $^{1} \cdot$ Massimo Tornatore $^{1}$
}

Received: date / Accepted: date

\begin{abstract}
The huge data demand envisioned for the $5 \mathrm{G}$ requires radically changes in the mobile network architecture and technology. Centralized Radio Access Network (C-RAN) is introduced as a novel mobile network architecture, designed to effectively support the challenging requirements of the future $5 \mathrm{G}$ mobile networks. In C-RAN, BaseBand Units (BBUs) are physically separated from their corresponding Radio Remote Heads (RRHs) and located in a central single physical location called BBU pool. The RRHs are connected to the BBU pool via the socalled fronthaul network. The "centralization" demonstrates remarkable benefits in terms of computational resources as well as power savings. Following this centralization, designing a survivable C-RAN becomes crucial as BBU pool and link failures might cause service outage for large number of users. In this paper, we propose three different approaches for the survivable BBU pool placement problem and traffic routing in C-RAN deployment over a 5G optical aggregation network. Namely we define the following protection scenarios: i) Dedicated Path protection, ii) Dedicated BBU protection and iii) Dedicated BBU and path protection. The three approaches are formalized as Integer Linear Programming (ILP) problems. The ILPs objectives are to minimize the number of BBU pools, the number of used wavelengths and the baseband processing computational resources, in terms of Giga operations Per Second (GOPS). We provide numerical results to compare the aforementioned protection strategies considering different network topologies. The results show the effect of the latency and the transport-network capacity on the BBU placement. We show the trade-off between the centralization degree and the tight latency requirements. Moreover, we discuss important insights about considering the different objective functions for each protection approach.
\end{abstract}

*Corresponding author

E-mail: mohamedkhaled.shehata@polimi.it

${ }^{1}$ Department of Electronics, Information and Bioengineering, Politecnico di Milano, Italy

${ }^{2}$ Arab Academy for Science and Technology and Maritime Transport, Cairo, Egypt

A preliminary version of this paper has been published in [1] 


\section{Introduction}

The design and operation of the upcoming $5 \mathrm{G}$ networks is expected to be challenged by very stringent constraints in terms of tolerable latency and required data rate [2], e.g., with end-to-end latency requirements below $1 \mathrm{~ms}$ for future $5 \mathrm{G}$ services [3]. As reported in [4] latency requirements for 5G applications varies from $100 \mathrm{~ms}$ to $1 \mathrm{~ms}$. To cope with the challenges envisioned for $5 \mathrm{G}$ mobile networks, including higher user data rate and reduced latency, one of the proposed network architectures is the so-called C-RAN [5]. The main idea of C-RAN is that multiple BBUs are placed in a single physical location (BBU pool), which is connected to the RRHs through a high capacity fronthaul network. Thanks to centralization, the baseband resources in the BBU pool can be virtualized and shared among several base stations; hence, a significant reduction in the overall computational resources can be achieved due to multiplexing gain. Moreover, BBU centralization allows to share maintenance costs and power consumption among several Base Stations (BSs) as well as promoting the utilization of advanced interference cancellation techniques as the Coordinated Multipoint.

Despite the advantages provided by C-RAN, the fronthaul network must be able to support very high bandwidth traffic with very low latency, leading to high transport-network cost. Optical aggregation networks based on Wavelength Division Multiplexing (WDM) are considered a relevant candidate solution to meet the fronthaul requirements [6]. In this context, an optimization placement of BBU pools in the aggregation network is crucial. The placement problem aims to choose a BBU pool location, which meets the latency and bandwidth requirements while maximizing the aforementioned centralization benefits. Many previous works have addressed the placement problem. The authors in [7] formulate an ILP model for the BBU pool placement problem to minimize the power of the aggregation network, while in [6] they investigate the amount of BBU consolidation achieved when using two different transport networks solutions (OTN and overlay) and jointly optimizing the BBUs and the electronic switches placement. Ref. [8] introduces a $\mathrm{BBU}$ placement problem for dense small cells over wireless fronthaul network and proposes a heuristic placement algorithm named SWAN to solve the problem. Ref. [9] formulates a Mixed Integer Linear Programming for Digital Unit (DU) pool placement optimization problem. The objective of the optimization problem is to minimize the total network cost. Similarly, ref. [10] proposes genetic algorithm to reduce the fronthauling cost through properly splitting and placing the baseband processing functions in the network.

An important aspect in the placement problem is how to deal with BBU pool and link failures, as these failures might cause service outage for a large area with a significant number of users. This has recently motivated novel research on the design of survivable C-RAN. Though survivability in the context of C-RAN and $5 \mathrm{G}$ has been rarely discussed in the literature, resilience aspects in the more general optical networks context has been widely investigated. Ref. [11] proposes a multipath protection scheme for data center services in an elastic optical networks based on the importance level of the services. Ref. [12] proposes a N:1 protection mechanism for Optical Line Terminals (OLTs) to minimize the number of backup OLTs required in a Passive Optical Network. Ref. [13] proposes a cost effective algorithm to minimize the cost of resilient flexible bandwidth optical networks. Ref. [14] presents two models for dedicated and shared path protection against a single 
link failure in elastic optical networks. Ref. [15] introduces an efficient restoration mechanisms to ensure service resilience in $5 \mathrm{G}$ cloud-based mobile network.

More related to our work, ref. [16] defines a protection problem for cloud radio access network against BBUs and link failures. The authors present different approaches based on $1+1$ dedicated path protection and $1+1$ virtual machine replication through an ILP algorithm, but the authors have not considered delay and link capacity constraints. Ref. [17] proposes a heuristic algorithm to connect each RRH to two BBU pools, primary and backup, while reducing the number of the backup BBU ports among the RRHs. Then in [18], the authors extend the work and formulate the problem as an ILP algorithm and compare it to the aforementioned heuristic algorithm. Ref. [19] formulates an ILP problem named cost-resilience BBU selection, where a mobile network operator has to select BBU equipment from several cloud providers with different failure probability and cost. The objective function minimizes the BBU pool processing power and maximizes the resiliency. So far, to the best of our knowledge no study has considered the latency and link capacity constraints for the fronthaul network in survivable BBU placement problem.

In [1] we present a survivable BBU placement algorithm where every RRH must be connected to two different BBU pools, one as a working (primary) pool and the second one as a backup pool and link protection is not considered. We formulate and solve the survivable BBU placement problem over an optical aggregation network using an Integer Linear Program (ILP).

As an extension of our work in [1], in this paper, we investigate the survivable BBU placement problem and traffic routing for $5 \mathrm{G}$ C-RAN deployment. We propose three protection approaches: i) Dedicated Path Protection (DPP) ii) Dedicated BBU Protection (DBP) and iii) Dedicated BBU and Path Protection (DBPP). When designing a resilient C-RAN, we formalize each approach as an ILP problem and solve it over a 5G optical aggregation network. For all these protection approaches we consider a multi objective optimization which minimizes: i) the number of BBU pools, ii) the number of used wavelengths and iii) the computational processing in terms of GOPS. Taking into consideration the maximum BBU pool computational processing and different network topologies with different connectivity we show how fronthaul latency requirements and links capacity contribute to the optimal solution. Moreover, we observe that minimizing the computational resources results in additional savings compared to minimizing the number of pools.

The remainder of this paper is organized as follows. Section 2 discusses the considerd C-RAN aspects (the network architecture and the BBU pool computational processing). Section 3 discusses the considered protection approaches. Section 4 shows the ILP formulations used to solve the resilient problems in the three protection scenarios. Section 5 shows the illustrative numerical results. Section 6 concludes the work.

\section{Centralized radio access network}

As already mentioned in Section 1, C-RAN introduces a significant computational, cost and power savings which come from the centralization of BBUs. Although the 


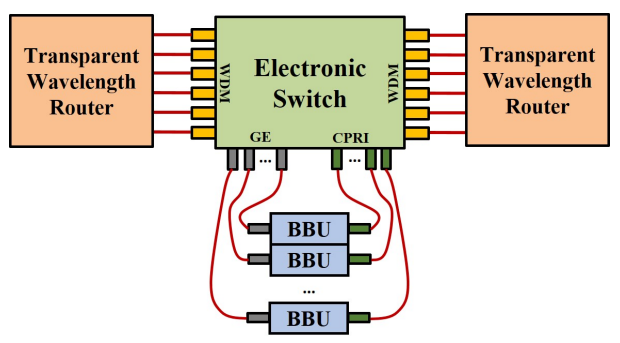

Fig. 1: BBU pool node architecture [6]

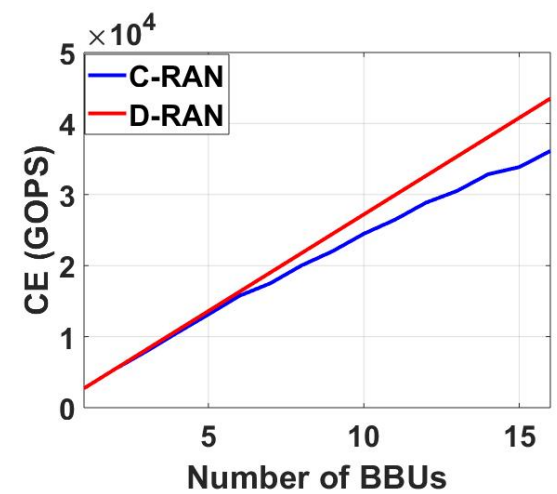

Fig. 2: Computational effort for different pools dimensions

aforementioned advantages, C-RAN requires a high-capacity and low-latency access/aggregation network to support fronthaul traffic. In the following, we will discuss the considered network architecture and the computational processing required by a BBU pool in terms of GOPS.

\subsection{Network architecture}

In this paper, we assume Opaque network architecture as in [7], where the BBU pool can be placed at any node. For intermediate nodes, this implies they become active and equipped with an electronic switch that terminates and aggregates all passing traffic, included traffic which is not destined to the hosted BBUs (i.e., all incoming lightpaths are terminated and processed by the switch). Hence, the main latency contributors considered in this work are the fiber propagation latency and the electronic switch latency. For the sake of simplicity, we assume that every $\mathrm{RRH}-\mathrm{BBU}$ connection (the so-called fronthaul connection) is transported over a dedicated wavelength. In this work we assume that there is a controller at each BBU pool and a centralized C-RAN controller on the top of them [20]. Controllers at the pools are responsible for detecting link and BBU failures. When a failure (i.e., BBU failure and/or link failure) is detected, the controller at pool report the C-RAN controller that can activate the backup BBU if needed and reconfigure the network. 
The architecture of a node hosting BBU pool is illustrated in Fig. 1. An electronic switch with WDM transport interfaces, Gigabit Ethernet (GE) interfaces and Common Public Radio Interfaces (CPRI) is depicted. The arrived lightpaths are multiplexed and demultiplexed by wavelength routers then forwarded to the switch via the WDM interfaces. Backhaul traffic destined to a hosted BBU is extracted and sent over the GE port, while fronthaul is collected by the switch from the CPRI interfaces, to be mapped into one or more output lightpaths.

\subsection{Baseband pool computational processing}

In this work, we consider the analytical model in [21] to calculate the computational processing of the BBU pool. This model is based on the complexity values estimated by the number of GOPS (what we call "Computational Effort" (CE) in this paper) for the baseband processing functions. We evaluate the computational effort through the following steps. First, we choose a statistical distribution (namely, normal or uniform) to model the spatial distribution of mobile users in a given serving area. Then we allocate the users to their serving cell sites according to a realistic user-cell site association strategy. After that, we apply a scheduling algorithm to distribute the physical resource blocks of the cell site among its users. Then, we calculate the computational effort (expressed in Giga Operations Per Second (GOPS)) per user after knowing channel condition, used resources, modulation, code rate and MIMO mode. Fig. 2 shows the required CE for the different pool dimensions and compare it to the CE needed by the same number of BBUs in the D-RAN case. In the D-RAN case, the CE scales linearly with number of BBUs, while in C-RAN, the higher number of BBUs per pool the higher savings in computational resources compared to D-RAN.

\section{Protection Scenarios}

To solve the survivable BBU placement problem we consider three different protection approaches as follows:

I) Dedicated Path Protection (DPP): This protection scheme provides resilience against link failure only, as shown in Fig. 3a. Consider BBU of the cell site at node "A" is hosted by the pool at node " $D$ " and routed over the path "AB-BD" as a primary path. In case of link failure, the connection will be routed over the backup path "AE-EF-FD". Note the link disjointness between the two paths.

II) Dedicated BBU Protection (DBP): The second protection scheme provides resilience against BBU failure only, as shown in Fig. 3b. Consider BBU of the cell site at node "A" is hosted by the pool at node "D" and routed over the path "AB-BD" as a primary path. In case of BBU failure the connection will be routed to the pool at node "C" through the path "AB-BC". In this approach, path disjointness between the primary and the backup pools is not considered. This protection scheme suits operators needs when failures occur in nodes with higher probability with respect to links.

III) Dedicated BBU and Path Protection (DBPP): The last protection scheme provides resilience against $\mathrm{BBU}$ and link failures, as shown in Fig. 


\section{Problem Formulation}

In this section we develop ILP formulations for DPP (ILP1), DBP (ILP2) and DBPP (ILP3) protection scenarios. We use a two-layer flow formulation, where an upper virtual layer is made up of virtual links, representing lightpaths originating and terminating in the nodes, and a lower layer consists of multi-fiber fronthaul links interconnecting the nodes. The survivable BBU pool placement problem is defined as follows:

- Given: network topology, number of wavelengths per link, maximum allowed fronthaul latency, and computational effort needed by a pool serving a given number of cell sites.

- ILP1 output: placement of BBUs such that each RRH is connected to one BBU pool through two link disjoint paths, one as a primary path and one as backup path.

- ILP2 output: placement of BBUs such that each RRH is connected to two BBU pools, one as a primary pool and one as a backup pool.

- ILP3 output: placement of BBUs such that each RRH is connected to one primary pool through primary path and one backup pool through backup path where the primary and backup paths are link disjoint.

- Objective: minimizing i) number of BBU pools, ii) number of wavelengths and iii) overall computational effort of all BBU pools. 
In the following we provide details of the three ILP formulations:

(A) Input sets and parameters:

a) $N$ is set of nodes in the physical network, $i, j, m, n \in N$.

b) $E_{p}$ is set of physical links, $i j \in E_{p}$.

c) $E_{v}$ is set of virtual links, $m n \in E_{v}$.

d) $d_{i j}$ is the propagation delay introduced by the physical link $i j$.

e) $d_{e s}$ is the latency introduced by the electronic switch at each node.

f) $D$ is the maximum allowable delay between cell site and the BBU pool (fronthaul delay).

g) $W$ is the number of wavelengths per each physical link.

h) $C_{q}$ is the computational effort in GOPS needed by a pool if it serves $q$ RRHs (the values of $C_{q}$ are given in Fig. 2).

i) $C$ is the maximum computational effort in GOPS that can be accommodated by a pool. Note that at most one pool can be accommodated in each node.

j) $M$ is a large number.

(B) Decision Variables:

a) $k_{i}=1$, if node $i \in N$ hosts a BBU pool (binary).

b) $b_{i, q}=1$, if the BBU pool hosted by node $i \in N$ serves $q$ RRHs as primary and backup (binary). If node $i$ does not host a pool, then $b_{i, 0}=1$.

c) $a_{i, m}=1$, if cell site at node $m \in N$ is assigned to a BBU pool at node $i \in N$ (binary) (employed only for ILP1).

d) $f_{i j}^{m n}=1$, if virtual link $m n \in E_{v}$ between the cell site at node $m \in N$ and the pool at node $n \in N$ is routed over the physical link $i j \in E_{p}$ as a primary path (binary) (employed only for ILP1).

e) $h_{i j}^{m n}=1$, if virtual link $m n \in E_{v}$ between the cell site at node $m \in N$ and the pool at node $n \in N$ is routed over the physical link $i j \in E_{p}$ as a backup path (binary) (employed only for ILP1).

f) $x_{i, m}=1$, if cell site at node $m \in N$ is assigned to a primary BBU pool at node $i \in N$ (binary) (employed only for ILP2 and ILP3).

g) $z_{j, m}=1$, if cell site $m \in N$ is assigned to a backup BBU pool at node $j \in N$ as a backup pool (binary) (employed only for ILP2 and ILP3).

h) $y_{i j}^{m n}=1$, if virtual link $m n \in E_{v}$ between the cell site at node $m \in N$ and the primary pool at node $n \in N$ is routed over the physical link ij $\in E_{p}$ (binary) (employed only for ILP2 and ILP3).

i) $t_{i j}^{m n}=1$, if virtual link $m n \in E_{v}$ between the cell site at node $m \in N$ and the backup pool at node $n \in N$ is routed over the physical link $i j \in E_{p}$ (binary) (employed only for ILP2 and ILP3).

(C) Objective function:

The multi-objective functions illustrated in Eqs. (1) and (2) are composed of three parts. The First term aims at minimizing the number of BBU pools. Second term minimizes the number of used wavelengths in the transport network. The last term minimizes the total computational effort required by the network.

I) ILP1

$$
\min \left(\alpha \sum_{i} k_{i}+\beta \sum_{i j} \sum_{m n}\left(f_{i j}^{m n}+h_{i j}^{m n}\right)+\gamma \sum_{i} \sum_{q} b_{i, q} C_{q}\right)
$$


II) ILP2 and ILP3

$$
\min \left(\alpha \sum_{i} k_{i}+\beta \sum_{i j} \sum_{m n}\left(y_{i j}^{m n}+t_{i j}^{m n}\right)+\gamma \sum_{i} \sum_{q} b_{i, q} C_{q}\right)
$$

Parameters $\alpha, \beta$ and $\gamma \in[0,1]$ can be tuned to select the primary objective of the optimization.

(D) Constraints:

I) ILP1

$$
\begin{aligned}
& \sum_{i} a_{i, m}=1, \quad \forall m \in N \\
& k_{i} \geq \frac{\sum_{m} a_{i, m}}{M}, \quad \forall m \in N \\
& \sum_{q} b_{i, q} C_{q} \leq C, \quad \forall i \in N \\
& \sum_{q} q b_{i, q}=\sum_{m} a_{i, m}, \quad \forall i \in N \\
& \sum_{q} b_{i, q}=1, \quad \forall i \in N \\
& \sum_{m n}\left(f_{i j}^{m n}+h_{i j}^{m n}\right) \leq W, \quad \forall i j \in E_{p} \\
& \sum_{i j} f_{i j}^{m n}\left(d_{i j}+d_{e s}\right)+d_{e s}\left(1-x_{m, m}\right) \leq D, \quad \forall m n \in E_{v}, \forall m \in N \\
& \sum_{i j} h_{i j}^{m n}\left(d_{i j}+d_{e s}\right)+d_{e s}\left(1-x_{m, m}\right) \leq D, \quad \forall m n \in E_{v}, \forall m \in N \\
& \sum_{j}\left(f_{i j}^{m n}-f_{j i}^{m n}\right)=\left\{\begin{array}{ll}
a_{n, i}, & \text { if } i=m, m \neq n . \\
-a_{i, m}, & \text { if } i=n, m \neq n . \\
0, & \text { otherwise. }
\end{array} \quad \forall m n \in E_{v}, \forall i \in N\right. \\
& \sum_{j}\left(h_{i j}^{m n}-h_{j i}^{m n}\right)=\left\{\begin{array}{ll}
a_{n, i}, & \text { if } i=m, m \neq n . \\
-a_{i, m}, & \text { if } i=n, m \neq n . \\
0, & \text { otherwise. }
\end{array} \quad \forall m n \in E_{v}, \forall i \in N\right. \\
& f_{i j}^{m n}+h_{i j}^{m n} \leq 1, \quad \forall m n \in E_{v}, \forall i j \in E_{p}
\end{aligned}
$$

Equation (3) enforces that each RRH is associated with exactly one BBU. Equation (4) is needed to identify BBU pools as the nodes which host at least one BBU. Equation (5) guarantees that GOPS of all the BBUs aggregated in certain pool does not exceed the maximum computational effort for that pool. Equations (6) and (7) are used to identify what is the computational complexity for the specific number of RRHs served by BBU pool $i$. Equation (8) guarantees that capacity of virtual links routed over a certain physical link does not exceed its capacity. Equations (9) and (10) 
ensure the latency requirements for the primary and backup paths, respectively. We take into account both the propagation delay $\left(d_{i j}\right)$ and the delay of the electronic switches $\left(d_{e s}\right)$, whose value is equal to the number of traversed links, plus one. The factor $\left(1-x_{m, m}\right)$ is necessary in order to disable this constraint in case the BBU is located at its cell site. Equations (11) and (12) are the flow constraints, which guarantee that all virtual links are mapped on a set of physical links for primary and backup paths, respectively. Equation (13) ensures the disjointness of the primary and backup paths.

II) ILP2

$$
\begin{aligned}
& \sum_{i} x_{i, m}=1, \quad \forall m \in N \\
& \sum_{i} z_{i, m}=1, \quad \forall m \in N \\
& x_{i, m}+z_{i, m} \leq 1, \quad \forall i \in N, \forall m \in N \\
& k_{i} \geq \frac{\sum_{m}\left(x_{i, m}+z_{i, m}\right)}{M}, \quad \forall i \in N \\
& \sum_{q} b_{i, q} C_{q} \leq C, \quad \forall i \in N \\
& \sum_{q} q b_{i, q}=\sum_{m}\left(x_{i, m}+z_{i, m}\right), \quad \forall i \in N \\
& \sum_{q} b_{i, q}=1, \quad \forall i \in N \\
& \sum_{m n}\left(y_{i j}^{m n}+t_{i j}^{m n}\right) \leq W, \quad \forall i j \in E_{p} \\
& \sum_{i j} y_{i j}^{m n}\left(d_{i j}+d_{e s}\right)+d_{e s}\left(1-x_{m, m}\right) \leq D, \quad \forall m n \in E_{v}, \forall m \in N \\
& \sum_{i j} t_{i j}^{m n}\left(d_{i j}+d_{e s}\right)+d_{e s}\left(1-x_{m, m}\right) \leq D, \quad \forall m n \in E_{v}, \forall m \in N \\
& \sum_{j}\left(y_{i j}^{m n}-y_{j i}^{m n}\right)=\left\{\begin{array}{ll}
x_{n, i}, & \text { if } i=m, m \neq n . \\
-x_{i, m}, & \text { if } i=n, m \neq n . \\
0, & \text { otherwise. }
\end{array} \quad \forall m n \in E_{v}, \forall i \in N\right. \\
& \sum_{j}\left(t_{i j}^{m n}-t_{j i}^{m n}\right)=\left\{\begin{array}{ll}
z_{n, i}, & \text { if } i=m, m \neq n . \\
-z_{i, m}, & \text { if } i=n, m \neq n . \\
0, & \text { otherwise. }
\end{array} \quad \forall m n \in E_{v}, \forall i \in N\right.
\end{aligned}
$$

Equations (14) and (15) enforce that each RRH is associated with exactly one primary BBU pool and one backup BBU pool. Equation (16) enforces primary BBU pool and backup BBU pool to be at different nodes for the same RRH. Equation (17) is needed to identify BBU pools as the nodes which host at least one (primary or backup) BBU. Equation (18) guarantees that GOPS of all the BBUs aggregated in certain pool does not exceed 
the maximum computational effort for that pool. Equations (19) and (20) ensure that the number of RRHs served by pool $i$ equals the sum of RRHs assigned to that pool $i$. Equation (21) guarantees that capacity of virtual links routed over a certain physical link does not exceed its capacity. Equations (22) and (23) ensure the latency requirements for the primary path and the backup path, respectively (similar to Eq. (9) and Eq. (10)). Equations (24) and (25) are the flow constraints, which guarantee that all virtual links connecting the RRHs with the primary BBU pools and backup BBU pools are mapped on a set of physical links.

III) ILP3

Consider all the constraints for ILP2 with applying the link disjointness between the path to the primary BBU pool and the path to the backup BBU pool as in Eq. (26). The cell site at node $m$ can use the physical link $i j$ only once (as a maximum) to route its traffic to any other node $n$ (where $n$ is a potential BBU pool). By this constraint, we can make sure that the physical link $i j$ is not used by the two outgoing paths of node $m$ (the first one to the primary BBU and the second one to the backup BBU) at the same time. As shown in Fig. 3c, the cell site at node "A" routes its traffic to two different BBU pools (pool at node "C" as a primary pool and pool at node "D" as a backup pool). For example, by applying Eq. (26), over physical link BC: $m=A, i j=B C, n=B, C, E, F, D$, this guarantees that only $y_{B C}^{A C}=1$. This means that the cell site at node "A" will use the physical link "BC" only once to route the traffic to the primary pool at node "C". Therefore, it (physical link "BC") will not be used to route the traffic of cell site at node "A" to backup pool at node "D" (link disjointness).

$$
\sum_{n}\left(y_{i j}^{m n}+t_{i j}^{m n}\right) \leq 1, \quad \forall m \in N, \forall i j \in E_{p}
$$

(E) Problem complexity

The total number of variables $N_{v a r}$ for all three optimization problems is obtained with the formula:

$$
N_{\text {var }}=|N|\left(1+3|N|+2\left|E_{p}\right|\left|E_{v}\right|\right)
$$

The number of constraints varies according to the scenario. In the case of DPP scenario, the number of constraints is given as:

$$
N_{D P \text { Pconst }}=|N|\left(5+4\left|E_{v}\right|\right)+\left|E_{p}\right|\left(1+\left|E_{v}\right|\right)
$$

Differently, in the DBP scenario, constraints complexity is:

$$
N_{D B \text { P const }}=|N|\left(6+|N|+4\left|E_{v}\right|\right)+\left|E_{p}\right|
$$

Finally, in the DBPP scenario, constraints complexity is:

$$
N_{D B P \text { Pconst }}=|N|\left(6+|N|+4\left|E_{v}\right|+\left|E_{p}\right|\right)+\left|E_{p}\right|
$$

For the number of variables $N_{v a r}$, we observe that the dominate term is $2 .|N| .\left|E_{p}\right| \cdot\left|E_{v}\right|$, while for the constraints complexity (for the different scenarios) the dominate term is $4 .|N| .\left|E_{v}\right|$. Therefore, the problem complexity for all the proposed protection scenario, given by the sum of the number of variables and the number of constraints, is in the order of $O\left(|N| \cdot\left|E_{v}\right| \cdot\left|E_{p}\right|\right)$. 


\section{Illustrative Numerical Results}

\subsection{Evaluation Settings}

In this work, we consider two different aggregation network topologies with different levels of node connectivity degree, as in [18] (i.e., ratio between the total number of edges and the total number of nodes in the network) as shown in Fig. 4. Each topology consists of 30 nodes uniformly distributed over a dense urban square region of $7.5 \mathrm{~km}^{2}$. For simplicity, we assume that every node contains one RRH. Every node represents a potential BBU pool. We consider a $20 \mu s$ latency for each electronic switch and one fiber per link, carrying $W=6$ wavelengths at $10 \mathrm{~Gb} / \mathrm{s}$. We assume that the pool can accommodate 20 BBUs as maximum with 44080 GOPS. To solve our optimizations we used ILOG CPLEX 12.0 on a workstation equipped with $8 \times 2 \mathrm{GHz}$ processors and $32 \mathrm{~GB}$ of RAM. In this section, we show the results for the following objective functions.

- First objective function (O1): we optimize the number of BBU pools as a first objective, then the number of used wavelengths as the second objective.

- Second objective function (O2): we first optimize the number of used wavelengths then the number of BBU pools.

- Third objective function (O3): we optimize first the computational effort, then the number of BBU pools, then the number of used wavelengths.

The values of the parameters $\alpha, \beta, \gamma$ to select the desired objective function are given in Table 1. Note that the number of wavelengths is counted as the summation of the variables $y_{i j}^{m n}$ and $t_{i j}^{m n}$ in DBP and DBPP scenarios and as summation of the variables $f_{i j}^{m n}$ and $h_{i j}^{m n}$ in DPP scenario.

\subsection{Numerical Results}

Fig. 5 shows the number of BBU pools and the number of used wavelengths (left and right bars, respectively) as a function of the maximum allowable fronthaul latency (D) considering the three protection approaches and objetive function $\mathrm{O} 1$ in network topology "a" (connectivity degree 1.13).

For DPP (Fig. 5a) at low latency values $(D \leq 50 \mu s)$ no centralization occurs. All BBUs are placed at the cell sites (no wavelengths utilized for the transport network) as the tight latency constraint does not allow the BBUs to be separated from the RRHs and to interconnect via two link-disjoint paths. To this end, the solution obtained is to distribute all the BBUs (place each BBU at its cell site); hence no links are established to perform link protection. As the latency value increases $(D=100 \mu s)$ only few BBUs can be centralized with the two disjoint paths

Table 1: Parameters to select the objective function

\begin{tabular}{l|lll}
\hline & $\alpha$ & $\beta$ & $\gamma$ \\
\hline O1 & 1 & $10^{-3}$ & 0 \\
O2 & $10^{-3}$ & 1 & 0 \\
O3 & 1 & $10^{-3}$ & 10 \\
\hline
\end{tabular}




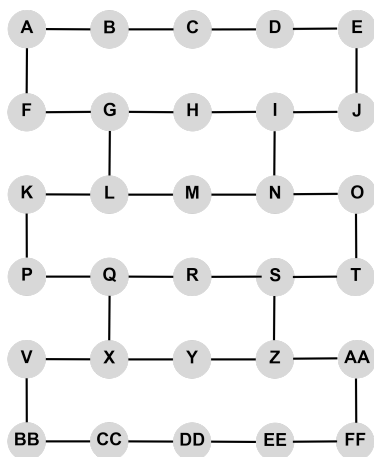

(a) Topology "a"

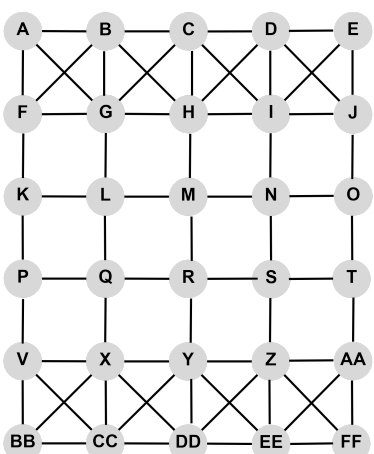

(b) Topology "b"

Fig. 4: Network Topologies

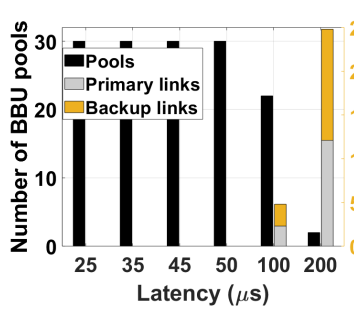

(a) DPP

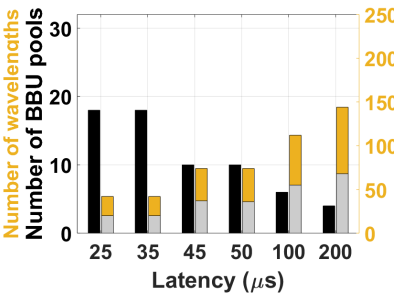

(b) DBP

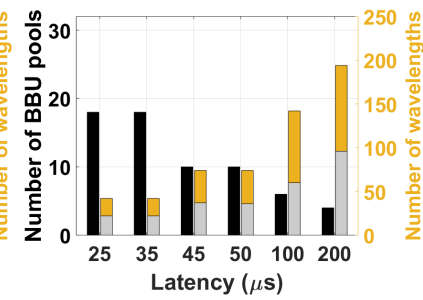

(c) DBPP

Fig. 5: Number of BBU pools and wavelengths (Topology = "a", Objective function $=\mathrm{O} 1$ )

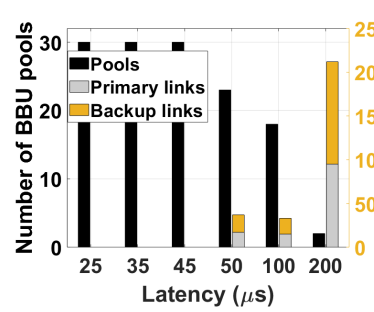

(a) DPP

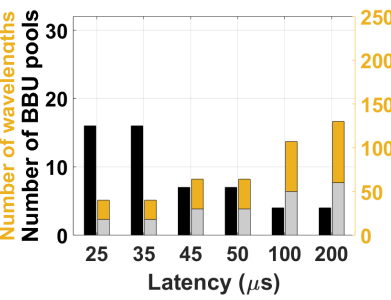

(b) DBP

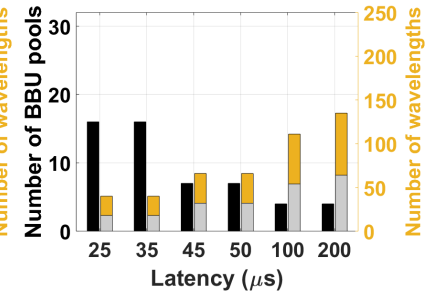

(c) DBPP

Fig. 6: Number of BBU pools and wavelengths (Topology = "b", Objective function $=\mathrm{O} 1)$

resulting in high number of BBU pools (22 BBU pools) and low number of utilized wavelengths (48 wavelengths). For more relaxed latency values $(D=200 \mu s)$, the number of BBU pools decreases (2 pools) while the number of wavelengths increases (250 wavelengths). Note that, the minimum number of pools can be achieved is 2 as one pool can accommodate up to 20 BBUs and the number RRHs in the network is 30 . 


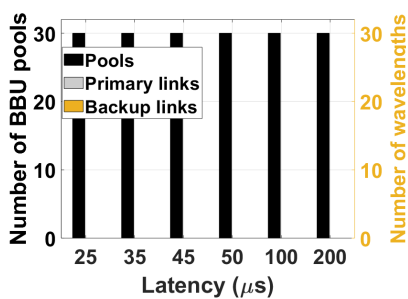

(a) DPP

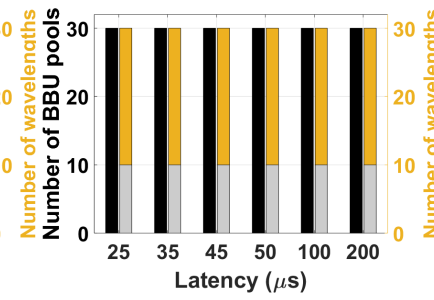

(b) DBP

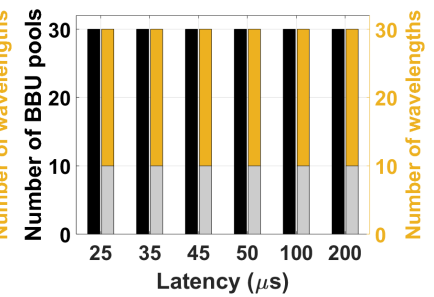

(c) DBPP

Fig. 7: Number of BBU pools and wavelengths (Topology = "a", Objective function $=\mathrm{O} 2$ )

For DBP and DBPP (Fig. 5b and Fig. 5c, respectively), no solution could be obtained for $D<25 \mu \mathrm{s}$, as the strict latency does not allow BBUs to be hosted outside the cell site; hence no BBU protection can be guaranteed in these cases. For low latency values $(D \leq 45 \mu s)$ most of the nodes (18 nodes) must be activated as pools. In this case, the tight latency values force one BBU (the primary or the backup) to be placed at the cell site while the other BBU is placed in one of the adjacent nodes ,resulting in low number of utilized wavelengths. As the maximum allowable latency increases, the number of BBU pools decreased until it reaches 4 pools at $D=200 \mu \mathrm{s}$ with the maximum number of utilized wavelengths.

By comparing the three protection approaches, DBP and DBPP have the same performance except for high latency values $(D \geq 100 \mu \mathrm{s})$, the number of utilized wavelengths in DBPP is higher by $35 \%$ as in DBPP path protection is considered. DPP does not provide centralization nor protection at tight latency values $(D<$ $100 \mu s)$. For latency value $D=100 \mu s$, DPP can be guaranteed with higher number of BBU pools (22 pools) compared to the two other scenarios (6 pools) although it does not provide BBU protection. This is due to the tight latency constraint on both the primary and backup paths to reach the same pool, forcing high number of BBUs to be placed at their cell sites; hence high number of nodes are activated as a $\mathrm{BBU}$ pool. One the other hand, in DBP and DBPP, meeting those latency values is applicable -with higher degree of centralization compared to DPP- as we route the connections to two disjoint pools (primary and backup), leading to less number of the pools. At high latency value $D=200 \mu s$, DPP has low number of BBU pools ( 2 pools) compared to DBP and DBPP (4 pools) as BBU protection is not considered in DPP. Subsequently, the number of utilized wavelengths in DPP (248 wavelengths) is higher than that in DBP and DBPP (144 and 194 wavelengths, respectively).

Fig. 6 shows the number of BBU pools and the number of used wavelengths (left and right bars, respectively) as a function of the maximum allowable fronthaul latency (D) considering the three different protection approaches and objective function O1 in network topology "b" (connectivity 2.16). It is clear that the results for topology "b" follow the same manner of topology "a". Unlike toplogy "a", DBP (Fig. 6b) and DBPP (Fig. 6c) have almost equal number wavelengths at all the latency values. This can be explained considering that as the connectivity is higher compared to topology "a", the disjointness of the primary and backup paths does not contribute to the number of utilized wavelengths. 


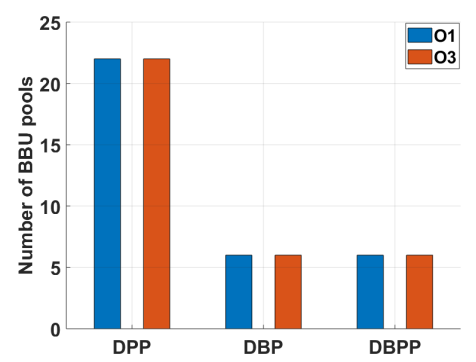

(a) Number of BBU pools Vs protection approach

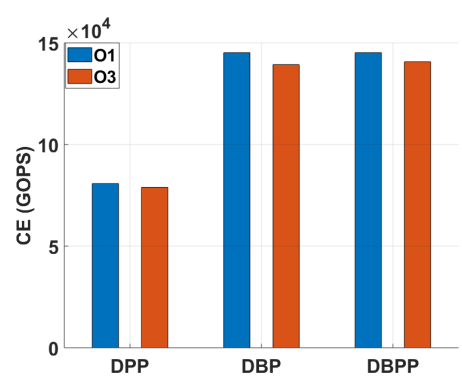

(b) Computational effort in GOPS Vs protection approach

Fig. 8: Number of BBU pools and the computational effort (Topology = "a", Objective function $=\mathrm{O} 1$ and $\mathrm{O} 3$ )

Fig. 7 shows the number of BBU pools and wavelengths as a function of the maximum allowable fronthaul latency (D) considering the three protection approaches and objective function O2 in network topology "a". For DPP (Fig. 7a), no centralization occurs, the BBUs are placed at the cell sites for all the latency values. This can be explained by that as minimizing the number of wavelengths is the first priority in $\mathrm{O} 2$ so the BBUs are placed at the cell site leading to zero utilized wavelengths, subsequently, no RRH-BBU connections to protect. For DBP (Fig. 7b) and DBPP (Fig. 7c), the number of BBU pools and number of used wavelengths are constant over the different latencies values. As the first objective of $\mathrm{O} 2$ is to minimize the number of wavelengths, the solver keeps one BBU (the primary or the backup) at the cell site and allocates the other BBU in the adjacent node leading to minimum as well as constant number of wavelengths. Note that we obtain the same results for topology "b", since in DPP all BBU located at the cell site so connectivity does not contribute. Similarly, for DBP and DBPP the $\mathrm{BBU}$ is placed at the adjacent node.

Fig. 8 compares objectives $\mathrm{O} 1$ and $\mathrm{O} 3$ in terms of the number of pools and the amount of computational effort considering topology "a" for $D=100 \mu \mathrm{s}$. Fig. 8a shows the number of BBU pools for $\mathrm{O} 1$ and $\mathrm{O} 3$ considering the three protection approaches. The results show that adding a term to minimize the computational effort does not contribute to the number of the BBU pools as no significant difference is observed between $\mathrm{O} 1$ and $\mathrm{O} 3$ for all the protection approaches. Fig. 8b shows the computational effort in GOPS corresponding to each objective function and protection approach. Despite O1 and O3 have the same number of BBU pools, the two objectives provide different amount of computational effort. While minimizing the GOPS in O3 not only guarantee the minimum number of pools but also choose combinations for the number of BBUs to be placed in the pools with higher multiplexing gain (less computational effort). By using O3 we estimate saving in computational effort up to $2.2 \%$ in DPP, $4.1 \%$ in DBP and $3.1 \%$ in DBPP with respect to $\mathrm{O} 1$. In general, for other network topologies, minimizing the computational effort might not give the minimum number of BBU pools although giving the lowest amount of GOPS. 


\section{Conclusion}

In this work, we have investigated the survivable C-RAN deployment problem over an optical aggregation network through three protection approaches namely: i) dedicated path protection ii) dedicated BBU protection iii) dedicated BBUand path protection. We have formulated optimization problem for each approach through an ILP model with different objectives, minimizing number of BBU pools, number of used wavelengths and overall computational effort. We show the results of the optimization problems for the three approaches on two different network topologies with different connectivity values considering latency, link capacity and the BBU pool computational resource capacity constraints. Finally, we show that minimizing the computational effort results in additional savings compared to the traditional minimization of the number of BBU pools.

As a future work, we will consider grooming and wavelength assignment features in the model. Moreover, we plan to develop a heuristic algorithm to deal with larger network instances.

Acknowledgements The research leading to these results has received funding from the European Community under grant agreement no. 761727 Metro-Haul project.

\section{References}

1. Shehata M, Ayoub O, Musumeci F, Tornatore M (2018) Survivable BBU Placement for C-RAN over Optical Aggregation Networks. In: 20th International Conference on Transparent Optical Networks (ICTON), IEEE, Romania, Bucharest, pp 1-4

2. Hossain E, Hasan M (2015) 5G cellular: Key enabling technologies and research challenges. IEEE Instrumentation \& Measurement Magazine 18(3):11-21

3. Simsek M, Aijaz A, Dohler M, Sachs J, Fettweis G (2016) 5G-Enabled Tactile Internet. Journal of Selected Areas in Communication 34(3):460-473

4. Schulz P, Matthe M, Klessig H, Simsek M, Fettweis G, Ansari J, Ashraf SA, Almeroth B, Voigt J, Riedel I, et al (2017) Latency critical IoT applications in $5 \mathrm{G}$ : Perspective on the design of radio interface and network architecture. IEEE Communications Magazine 55(2):70-78

5. Checko A, Christiansen HL, Yan Y, Scolari L, Kardaras G, Berger MS, Dittmann L (2015) Cloud RAN for mobile networks- A technology overview. IEEE Communications surveys \& tutorials 17(1):405-426

6. Musumeci F, Bellanzon C, Carapellese N, Tornatore M, Pattavina A, Gosselin S (2016) Optimal BBU placement for 5G C-RAN deployment over WDM aggregation networks. Journal of Lightwave Technology 34(8):1963-1970

7. Carapellese N, Tornatore M, Pattavina A (2014) Energy-efficient baseband unit placement in a fixed/mobile converged WDM aggregation network. IEEE Journal on Selected Areas in Communications 32(8):1542-1551

8. Riggio R, Harutyunyan D, Bradai A, Kuklinski S, Ahmed T (2016) SWAN: Base-band units placement over reconfigurable wireless front-hauls. In: 12th International Conference on Network and Service Management (CNSM), IEEE, Montreal, Canada, pp 28-36 
9. Lisi SS, Alabbasi A, Tornatore M, Cavdar C (2017) Cost-effective migration towards C-RAN with optimal fronthaul design. In: IEEE International Conference on Communications (ICC), IEEE, Paris, France, pp 1-7

10. Liu J, Zhou S, Gong J, Niu Z, Xu S (2015) Graph-based framework for flexible baseband function splitting and placement in C-RAN. In: IEEE International Conference on Communications (ICC), IEEE, London, UK, pp 1958-1963

11. Yang H, Cheng L, Yuan J, Zhang J, Zhao Y, Lee Y (2015) Multipath protection for data center services in openflow-based software defined elastic optical networks. Optical Fiber Technology 23:108-115

12. Nag A, Payne DB, Ruffini M (2016) N:1 protection design for minimizing OLTs in resilient dual-homed long-reach passive optical network. IEEE/OSA Journal of Optical Communications and Networking 8(2):93-99

13. Chen B, Zhang J, Xie W, Jue JP, Zhao Y, Shen G (2016) Cost-effective survivable virtual optical network mapping in flexible bandwidth optical networks. Journal of Lightwave Technology 34(10):2398-2412

14. Tomassilli A, Jaumard B, Giroire F (2018) Path protection in optical flexible networks with distance-adaptive modulation formats. In: 22nd International Conference on Optical Network Design and Modeling (ONDM), IEEE, Dublin, Ireland, pp 30-35

15. Taleb T, Ksentini A, Sericola B (2016) On service resilience in cloud-native $5 \mathrm{G}$ mobile systems. IEEE Journal on Selected Areas in Communications 34(3):483-496

16. Colman-Meixner C, Figueiredo GB, Fiorani M, Tornatore M, Mukherjee B (2016) Resilient cloud network mapping with virtualized BBU placement for cloud-RAN. In: International Conference on Advanced Networks and Telecommunications Systems (ANTS), IEEE, Bangalore, India, pp 1-3

17. Khorsandi BM, Raffaelli C, Fiorani M, Wosinska L, Monti P (2017) Survivable BBU Hotel placement in a C-RAN with an Optical WDM Transport. In: 13th International Conference of Design of Reliable Communication Networks (DRCN), VDE, Munich, Germany, pp 1-6

18. Khorsandi BM, Tonini F, Raffaelli C (2018) Design Methodologies and Algorithms for Survivable C-RAN. In: 22nd International Conference on Optical Network Design and Modeling (ONDM), IEEE, Dublin, Ireland, pp 106-111

19. Lyazidi MY, Giupponi L, Mangues-Bafalluy J, Aitsaadi N, Langar R (2017) A Novel Optimization Framework for C-RAN BBU Selection based on Resiliency and Price. In: 86th Vehicular Technology Conference (VTC-Fall), IEEE, Toronto, Canada, pp 1-5

20. Gifre L, Ruiz M, Castro A, Proietti R, Yoo SJB, Velasco L (2017) Experimental assessment of degradation-triggered reconfiguration in optically interconnected cloud-RAN. In: Optical Fiber Communication Conference, Optical Society of America, pp W2A-30

21. Shehata M, Elbanna A, Musumeci F, Tornatore M (2018) Multiplexing Gain and Processing Savings of 5G Radio-Access-Network Functional Splits. IEEE Transactions on Green Communications and Networking 2(4):982-991 